\title{
Probing the attentional control theory in social anxiety: An emotional saccade task
}

\author{
Matthias J. Wieser, Paul Pauli, and Andreas Mühlberger \\ University of Würzburg, Würzburg, Germany
}

\begin{abstract}
Volitional attentional control has been found to rely on prefrontal neuronal circuits. According to the attentional control theory of anxiety, impairment in the volitional control of attention is a prominent feature in anxiety disorders. The present study investigated this assumption in socially anxious individuals using an emotional saccade task with facial expressions (happy, angry, fearful, sad, neutral). The gaze behavior of participants was recorded during the emotional saccade task, in which participants performed either pro- or antisaccades in response to peripherally presented facial expressions. The results show that socially anxious persons have difficulties in inhibiting themselves to reflexively attend to facial expressions: They made more erratic prosaccades to all facial expressions when an antisaccade was required. Thus, these findings indicate impaired attentional control in social anxiety. Overall, the present study shows a deficit of socially anxious individuals in attentional control-for example, in inhibiting the reflexive orienting to neutral as well as to emotional facial expressions. This result may be due to a dysfunction in the prefrontal areas being involved in attentional control.
\end{abstract}

In anxiety disorders, there is a great deal of research in support of an association between anxiety and increased attentional bias toward threatening stimuli (e.g., angry faces in social anxiety) - a phenomenon that is called hypervigilance for threat (for an extensive review, see Bar-Haim, Lamy, Pergamin, Bakermans-Kranenburg, \& van Ijzendoorn, 2007). This attentional bias might be due to a lack of voluntary control over attention allocation. Accordingly, anxious persons might be impaired to inhibit the reflexive orienting to threatening stimuli by volition. In recent neuroimaging studies, it has been shown that anxiety selectively facilitates the early processing of threat stimuli and enhances distractibility toward taskirrelevant stimuli. This bias in threat processing is associated with enhanced amygdala activity and reduced activity in prefrontal cortical areas (especially the dorsal lateral prefrontal cortex [DLPFC] and the ventral lateral prefrontal cortex [VLPFC]), which are involved in top-down regulation of attention (Bishop, 2007; Bishop, Duncan, Brett, \& Lawrence, 2004). According to Bishop, this reduced recruitment of prefrontal areas indicates a diminished ability in highly anxious individuals to recruit these areas to augment attentional control.

From a cognitive point of view, M. W. Eysenck and colleagues (M. W. Eysenck, Derakshan, Santos, \& Calvo, 2007) put forward the attentional control theory of anxiety. According to this theory, it is assumed that anxious individuals show impairments in the efficient functioning of the goal-directed attentional system, which leads to an increased extent to which processing is influenced by the stimulus-driven attentional system. This assump- tion is made on the basis of the distinction between two attentional systems and the disruption of the balance between these two systems: the goal-oriented (involved in top-down control, influenced by current goals) and the stimulus-driven (involved in bottom-up control, influenced by salience of environmental input) systems. The former system is similar and functionally equivalent to the anterior attentional system that was proposed by Posner and Petersen (1990), which is assumed to be located in the prefrontal cortex. The latter is similar to the posterior attentional system that was also proposed by Posner and Petersen, which is located in temporo-parietal and ventral frontal areas of the brain. These two systems are generally thought to interact in their functioning (Pashler, Johnston, \& Ruthruff, 2001), whereas in anxiety disorders it is assumed that there is an imbalance that is due to an increase of the influence of stimulus-driven over goal-directed processes. Such an imbalance reduces inhibitory control of attention toward task-irrelevant stimuli, especially in a situation including salient or conspicuous distractors.

The antisaccade task is a well-established research tool to investigate executive functions such as the volitional control of attention in humans (see, e.g., Broerse, Crawford, \& den Boer, 2001). During this task, a fixation point is presented. Afterward, peripheral cues are randomly presented to the left or right of the fixation. Subjects are instructed to look either on the peripheral cue (prosaccade) or straight at its mirror-image position, which means at the opposite direction (antisaccade) (Hallett, 1978). Because of the reflexive-response (stimulus-driven) character of prosaccades triggered by 
the onset of a peripheral stimulus, they are not likely to be under the control of executive functions. In contrast, antisaccades require two processes: first, the inhibition of a prosaccade, and second, the volitional programming of a saccade in the opposite direction (Bell, Everling, \& Munoz, 2000). Subjects make errors in the antisaccade task-for example, looking at the peripheral stimulus involuntarily, despite being told to look at the opposite location (prosaccade error). Several groups of psychiatric and neurological patients show increased error rates in the antisaccade task as compared with healthy subjects (Everling \& Fischer, 1998; Hutton \& Ettinger, 2006; Reuter \& Kathmann, 2004). These increased error rates are usually interpreted as an "inhibition deficit" (e.g., Crawford, Bennett, Lekwuwa, Shaunak, \& Deakin, 2002; Everling \& Fischer, 1998).

In a recent neuroimaging study, Ettinger et al. (2008) identified the neural substrates of attentional control in an antisaccade paradigm. They found enhanced activation in the supramarginal gyrus, the DLPFC, and the VPFLC when participants successfully performed antisaccades. These activations are interpreted as being involved in the cognitive control and inhibition of the reflexive prosaccades. As was mentioned previously, such areas are also considered to play a substantial role in the top-down modulation of emotional processing in anxiety (Bishop, 2007).

The antisaccade task has been used to investigate the conflicts between reflexive and volitional behavioral responses in a variety of psychiatric disorders, including depression, anxiety disorders, and attention deficit hyperactivity disorder (ADHD). All of these studies used neutral stimuli, which do not attract attention by their meaning but by their mere appearance. In a recent study, it was found that depressed or anxious participants' accuracy in performance was less enhanced by reward contingencies than was that of healthy controls (Jazbec, McClure, Hardin, Pine, \& Ernst, 2005). ADHD patients were found to be impaired in performing correct antisaccades, indicating impaired executive functioning (Klein, Raschke, \& Brandenbusch, 2003).

Since the antisaccade performance reflects the ability to volitionally inhibit automatic processes - for example, to overwrite bottom-up processes by top-down controlperformance seems to be particularly modulated by emotionally significant stimuli such as emotional pictures or emotional facial expressions (Kissler \& Keil, 2008). Thus, an emotional adaption of this task might be a promising tool for investigating how emotional pictures capture attention and consequently have an impact on basic saccadic reactions (Kissler \& Keil, 2008). In the Kissler and Keil study, it was found that more antisaccade errors toward emotional (pleasant and unpleasant) than toward neutral IAPS pictures were made, which may lead to the conclusion that emotional stimuli have a considerable impact on saccadic reactions. This may be even more prominent in clinical populations, as was mentioned previously. Especially in anxiety disorders, a high impact of emotional (i.e., threatening) stimuli on gaze behavior is to be expected. According to the attentional control theory, these stimuli might lead to a reduced volitional inhibition of reflexive orienting toward them.

Indeed, recent studies have found evidence for fearrelevant emotional content influencing ocular motor behavior during visual search in spider-phobic participants (Gerdes, Alpers, \& Pauli, 2008; Miltner, Krieschel, Hecht, Trippe, \& Weiss, 2004; Rinck \& Becker, 2006). In all of these studies, phobic patients were distracted from ongoing tasks by abruptly appearing task-irrelevant (phobic) cues, which is in line with the predictions of the attentional control theory. Additionally, Gerdes and colleagues showed cue generalization: Erroneous fixation of the distractor stimulus was more pronounced in spider-phobic patients - even if these distractor stimuli contained flowers, mushrooms, or empty circles. Recently, the attention control theory was tested in trait anxiety using the antisaccade task (Derakshan, Ansari, Hansard, Shoker, \& Eysenck, 2009). Using facial expressions, it was found that high-anxious individuals showed longer antisaccade latencies than did low-anxious individuals; furthermore, this effect was greater for threatening than for happy or neutral faces. However, groups did not differ in terms of error rate in the antisaccade task.

In social phobia, the emotional expressions in a human face are regarded to be of special relevance. Numerous studies have shown that social-phobic individuals tend to judge all facial expressions as being more negative (see, e.g., Coles, Heimberg, \& Schofield, 2008), that they scan faces with a different pattern of eye movements (Horley, Williams, Gonsalvez, \& Gordon, 2003), that they show attentional biases for negative and positive emotional expressions (e.g., Garner, Mogg, \& Bradley, 2006b; Wieser, Pauli, Weyers, Alpers, \& Mühlberger, 2009), and that they show enhanced visual attention to angry faces (Kolassa, Kolassa, Musial, \& Miltner, 2007; Kolassa \& Miltner, 2006; Mühlberger et al., 2009). Moreover, socially anxious individuals seem to exhibit a general hypervigilance for facial expressions (Kolassa et al., 2007). These results are accompanied by neuroimaging studies that have revealed that socially anxious individuals - in comparison with controls - showed enhanced activations in emotional and face-specific areas (amygdala, insula, superior temporal sulcus) in response to facial expressions (happy, angry, fearful, and neutral) (Birbaumer et al., 1998; Gentili et al., 2008; Straube, Mentzel, \& Miltner, 2005). These results show that in socially anxious individuals, facial expressions per se lead to strong activations in areas related to emotional responses. As the findings of Gentili et al. showed, this is also accompanied by a decreased activation in prefrontal areas, which points at weakened attentional control and might be the reason for hypervigilance and abnormal scanning of faces in social anxiety.

To sum up, there is evidence that socially anxious individuals lack the volitional inhibition of automatic orienting to task-irrelevant stimuli such as emotional faces, or even faces in general. From a neurobiological perspective, the involvement of a neural network associated with face and emotion processing is considered to be critical in the development and maintenance of attentional dys- 
functions in social phobia. This neural architecture includes the responses in a threat-detection module that signifies the amygdala response to the visual presentation of threat, and the interaction of this system with areas generally involved in visual attention (extrastriate areas) and attentional control (anterior cingulate cortex [ACC], DLPFC, and VLPFC). Because of the assumed involvement of these prefrontal structures in potential regulation processes in emotion processing and in attentional control as measured by correct antisaccade performance, the antisaccade task seems to be an excellent tool to investigate the prefrontal-limbic emotional network, even without measuring underlying brain functions directly. Doing this might shed further light on the attentional dysfunctions in social phobia, the meaning of facial expressions in social phobia, and the underlying neural architecture.

The antisaccade task has been used to investigate the conflicts between reflexive and volitional behavioral responses in a variety of psychiatric disorders. All of these studies used neutral stimuli that do not attract attention by their meaning but by their mere appearance. Because of the fact that incorrect responses and saccade latencies during the antisaccade task reflect the ability to volitionally inhibit automatic processes, these measures seem to be particularly modulated by emotionally and motivationally significant stimuli such as emotional pictures or emotional facial expressions (Kissler \& Keil, 2008).

The present study examined the attentional processes in socially anxious persons in response to emotional facial expressions by means of eyetracking in an emotional saccade task. Because threatening (i.e., angry) as well as other negative facial expressions are supposed to be meaningful for socially anxious persons, sad and fearful facial expressions were included in this study in addition to angry, happy, and neutral expressions.

As was previously shown in several antisaccade task studies, we expected to replicate the findings of prolonged latencies of correct antisaccades in comparison with correct prosaccades, along with a higher error rate in the antisaccade than in the prosaccade task. On the basis of the literature on attentional biases toward facial stimuli in so- cial anxiety, and the predictions of the attentional control theory, we expected high socially anxious participants to make more errors and to show longer latencies in response to angry facial expressions in the antisaccade task, in contrast with shorter latencies in response to angry faces in the prosaccade task. Because of the findings of enhanced visual attention to other facial expressions in social phobia, we also expected to find influences on the antisaccade performance. Altogether, the present study aimed at a further elucidation of attentional processes and their volitional control in social anxiety.

\section{METHOD}

\section{Participants}

A total of 180 students of the University of Würzburg were screened for social anxiety by means of the German version of the Brief Fear of Negative Evaluation Scale (BFNE; Leary, 1983). Persons within the lowest $20 \%$ (referred to as "low socially anxious," LSA) scores, the highest $20 \%$ (referred to as "high socially anxious," HSA) scores, or in the range from $40 \%$ to $60 \%$ (medium socially anxious, referred to as "controls") were invited to take part in the study. Since men as well as women were recruited and differences in mean sum scores were to be expected (Turk et al., 1998), these distributions were made separately for each sex. The cut-offs for women were below 33.0 for LSA, between 38.0 and 42.0 for controls, and above 46.0 for HSA; the cut-offs for men were below 27.8 for LSA, between 32.8 and 39.0 for controls, and above 41.0 for HSA. Before the experimental tasks, the participants completed the German version of the Social Phobia and Anxiety Inventory (SPAI; Fydrich, 2002; Turner, Beidel, Dancu, \& Stanley, 1989), the BFNE once more, the German version of the State-Trait Anxiety Inventory (STAI; Laux, Glanzmann, Schaffner, \& Spielberger, 1981; Spielberger, Gorsuch, \& Lushene, 1970), the German version of the Positive and Negative Affect Scale (PANAS; Krohne, Egloff, Kohlmann, \& Tausch, 1996; Watson, Clark, \& Tellegen, 1988), the German version of the Eysenck Personality Questionnaire (EPQRK; H. J. Eysenck \& S. B. G. Eysenck, 1991; Ruch, 1999), and a questionnaire on sociodemographic variables.

Altogether, 21 LSA, 20 control, and 21 HSA participants completed the experimental session. The participants' ages ranged from 19 to 42 years, and 49 (80.1\%) of them were female. Men and women were equally distributed to the three groups of social anxiety $\left[\chi^{2}(2\right.$, $N=61)<1, p=.99]$. Mean age and mean questionnaire scores of the three groups are shown in Table 1. The three groups differed (as was

Table 1

Characteristics for Each Group

\begin{tabular}{|c|c|c|c|c|c|c|c|c|}
\hline \multirow[b]{2}{*}{ Variable } & \multicolumn{2}{|c|}{ LSA } & \multicolumn{2}{|c|}{ Controls } & \multicolumn{2}{|c|}{ HSA } & \multirow[b]{2}{*}{$F(2,58)$} & \multirow[b]{2}{*}{$p$} \\
\hline & $M$ & $S D$ & $M$ & $S D$ & $M$ & $S D$ & & \\
\hline SPAI & 54.9 & 18.9 & 65.6 & 21.8 & 83.7 & 28.8 & 7.7 & .001 \\
\hline BFNE & 28.6 & 5.1 & 38.1 & 5.1 & 46.5 & 6.4 & 51.8 & .001 \\
\hline STAI-trait & 34.3 & 6.4 & 37.9 & 7.1 & 49.0 & 10.4 & 18.7 & .001 \\
\hline STAI-state & 34.7 & 4.8 & 35.1 & 5.3 & 41.0 & 8.5 & 6.2 & .004 \\
\hline PANAS-PA & 25.9 & 7.2 & 25.0 & 6.8 & 24.2 & 6.9 & 0.3 & .755 \\
\hline PANAS-NA & 11.0 & 2.1 & 11.3 & 1.3 & 13.9 & 4.1 & 7.1 & .002 \\
\hline EPQ (psychoticism) & 3.8 & 1.7 & 2.8 & 1.3 & 3.2 & 2.4 & 1.5 & .230 \\
\hline EPQ (neuroticism) & 3.1 & 1.8 & 4.3 & 2.6 & 7.1 & 2.8 & 14.3 & .001 \\
\hline EPQ (extraversion) & 8.7 & 3.1 & 9.0 & 3.0 & 7.2 & 3.2 & 2.0 & .143 \\
\hline EPQ (social desirability) & 1.8 & 1.5 & 1.7 & 1.7 & 1.7 & 1.8 & 0.2 & .979 \\
\hline Age & 23.0 & 5.1 & 22.2 & 2.3 & 21.9 & 2.0 & 0.7 & .545 \\
\hline
\end{tabular}

Note-Male to female gender ratio $=4: 16$ for low-social-anxiety (LSA) group, 4:17 for controls, and 4:16 for high-social-anxiety (HSA) group. SPAI, Social Phobia and Anxiety Inventory; BFNE, Brief Fear of Negative Evaluation Scale; STAI, State-Trait Anxiety Inventory; PANAS, Positive and Negative Affect Scale (PA, positive affect; NA, negative affect); EPQ, Eysenck Personality Questionnaire (short form). 
expected) in their scores of social anxiety as measured by the SPAI $\left[F(2,58)=8.9, p<.001, \eta_{\mathrm{p}}^{2}=.25\right]$ and the $\operatorname{BFNE}[F(2,58)=51.5$, $\left.p<.001, \eta_{\mathrm{p}}^{2}=.65\right]$, and in trait anxiety as measured by the STAI-trait part $\left[F(2,58)=18.7, p<.001, \eta_{\mathrm{p}}^{2}=.40\right]$. Furthermore, the groups reported different levels of actual anxiety as measured by the STAI-state part $\left[F(2,58)=6.3, p=.004, \eta_{\mathrm{p}}^{2}=.19\right]$. No differences were found in the positive subscales of the PANAS $(p>.50)$ or in mean age $(p>$ $.50)$, whereas significant effects were found for the PANAS subscale of negative affect $\left[F(2,58)=6.4, p=.003, \eta_{\mathrm{p}}^{2}=.19\right]$. Furthermore, groups differed significantly in their levels of neuroticism $[F(2,58)=$ $\left.14.7, p<.001, \eta_{\mathrm{p}}^{2}=.34\right]$, as measured by the EPQ. None of the 61 final participants had either psychiatric or neurological disorders, and they reported normal or corrected-to-normal vision. All of them gave their informed consent and received course credits or a payment of $€ 12$ for their participation. The protocol for the study was approved by the ethics committee of the Deutsche Gesellschaft für Psychologie (German Association of Psychology).

\section{Stimulus Materials and Apparatus}

Facial expressions of anger, joy, sadness, and fear, along with corresponding neutral facial expressions, served as visual stimuli. These facial expressions were created by using Poser 5 animation software (Curious Labs, Santa Cruz, CA). This software allows creating computer-generated facial expressions, in an easy and controlled way. Spencer-Smith et al. (2001) offered a software extension to manipulate action units separately according to the facial action coding system (Ekman \& Friesen, 1978). Notably, Spencer-Smith et al. could show that ratings of quality and the intensity of the avatars' emotional expressions were comparable to human expressions from the Pictures of Facial Affect (Ekman \& Friesen, 1976). The emotional facial expressions were created out of a prototype female and male face (for details, see Spencer-Smith et al., 2001). Each emotional expression was combined with six different styles (hairstyles and hair colors) for the female and the male faces, so that altogether, 60 different stimuli were created ( 5 emotional expressions $\times$ 6 types $\times 2$ sexes; for examples, see Figure 1$)$.
In the experimental task, participants had to perform a pro- and an antisaccade task in response to the facial expressions. At first, a fixation cross was presented $(500 \mathrm{msec})$, followed by the instruction $(500 \mathrm{msec})$ to perform a prosaccade ("Hin!" ["Toward!']) or an antisaccade ("Weg!" ["Away!"]) to or away from the appearing picture. Afterward, the facial expressions were presented for $1,000 \mathrm{msec}$ in the left or the right peripheral field of vision without gap. The center of the picture was located $8.9 \mathrm{~cm}$ to the left or right of the fixation cross $\left(10.1^{\circ}\right.$ of visual angle). Pictures were $13.6 \mathrm{~cm}$ high and $10 \mathrm{~cm}$ wide $\left(15.2^{\circ}\right.$ and $11.3^{\circ}$ of visual angle, respectively). The 60 facial expressions were presented once on the right side and once on the left side, which means that there were 120 trials in total. The pictures were presented on a dark gray background on a 17-in. monitor that had a resolution of $1,024 \times 768$ pixels. Instructions were balanced concerning picture content and location (left vs. right). The presentation order was random for each participant.

After the tasks, the participants viewed each facial expression once again and were asked to rate each picture on 9-point Likert scales referring to arousal and valence. Scales ranged from $1=$ very unpleasant to $9=$ very pleasant for the valence rating, and from $1=$ not arousing at all to $9=$ very arousing for the arousal ratings.

During the task, the participants' eye movements were monitored continuously by a remote eyetracking recording system (iView X Hi-Speed, SensoMotoric Instruments, Berlin, Germany). Eyemovement measures were based on the corneal reflection caused by an infrared light source (energy $3.5 \mathrm{~mW} / \mathrm{cm}^{2}$ at a distance of $100 \mathrm{~cm}$ ). Movements of the right pupil were recorded continuously at $240 \mathrm{~Hz}$ while subjects were performing the task. The eyetracking system included a chin- and forehead rest to minimize head movements and to ensure a distance of $50 \mathrm{~cm}$ between the participant's eyes and the center of the display screen. Before each experiment, a standardized calibration procedure for eye movements was performed by asking the participant to focus on 13 yellow crosshairs inserted on the screen's display space in random order. Within an experimental trial, the facial expressions were presented only when the subject focused exactly on the fixation cross, ensuring that
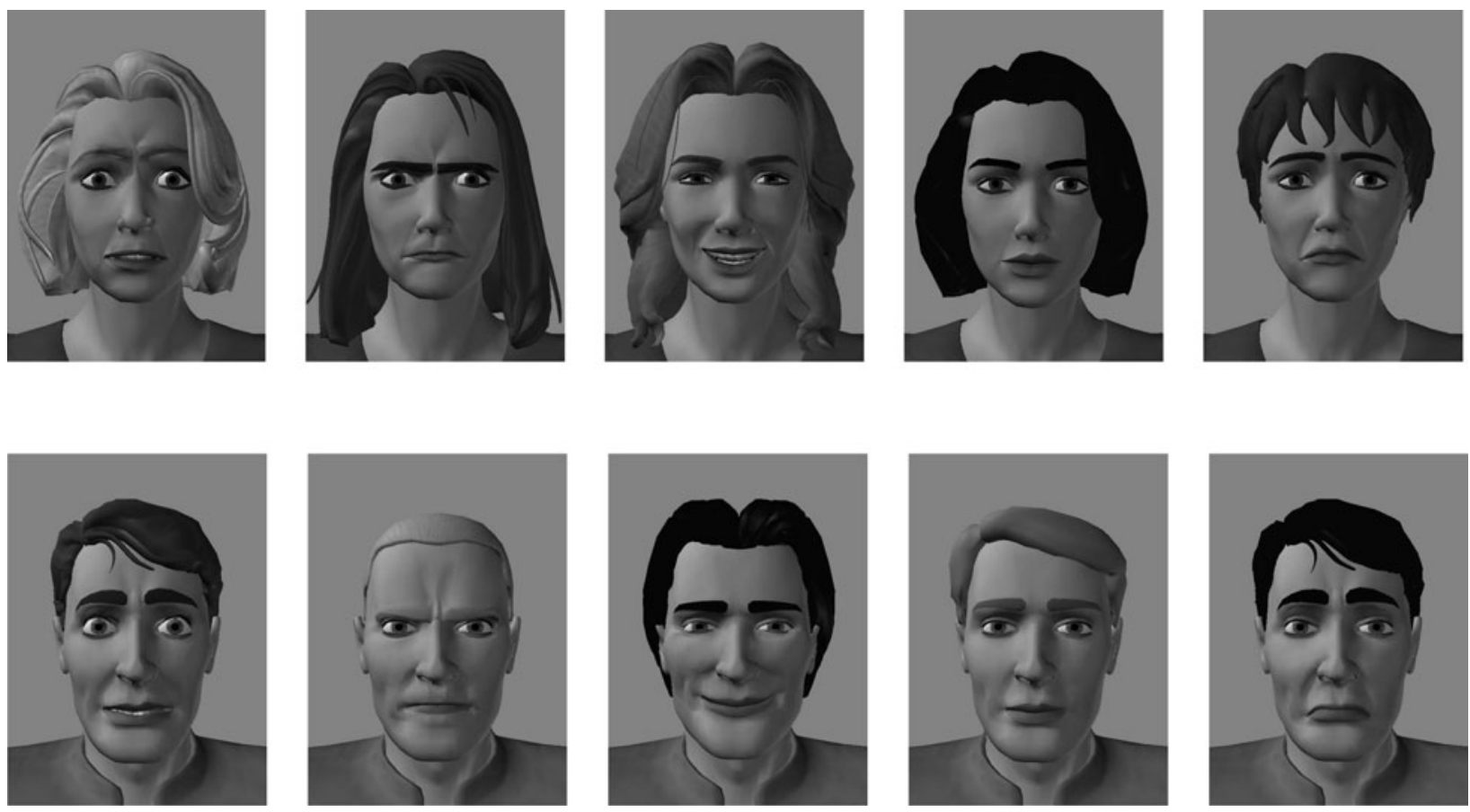

Figure 1. Examples of the five facial expressions. Female faces are in the upper row; male faces are in the lower row. Facial expressions from left to right: fearful, angry, happy, neutral, and sad. 
the initial position of the retina was controlled across all participants during each trial. This was achieved by defining a circular area with a diameter of $1 \mathrm{~cm}$ around the center of the fixation cross. The presentation of the pictures started only when the participants' gaze matched the spatial coordinates of this circle for at least $500 \mathrm{msec}$.

\section{Procedure}

After arriving at the laboratory, participants completed the informed consent form and the questionnaires described previously. Then, they were comfortably seated in a chair in front of the monitor. Head movements were restrained by the chin and forehead rest. Afterward, participants were instructed to follow the instructions presented on the screen. In the first part of the experiment, after the 13-point calibration procedure, participants performed a free viewing task while watching two simultaneously presented faces. The results of this task are reported elsewhere. Then, after another 13-point calibration procedure, the experimental session started with practice trials of the saccade task (altogether 10 trials) in which the pro- and the antisaccade task was explained. When participants reported that they had understood the task and the experimenter had confirmed this by visual inspection of the task performance, the experimental session was continued with 120 trials of the pro- and antisaccade task. Each trial started with a fixation cross that had to be fixated for at least $500 \mathrm{msec}$. Afterward, the instructions "towards" and "away" appeared for $500 \mathrm{msec}$ just above the fixation cross, followed by the target picture appearing without a gap for $1 \mathrm{sec}$ at the left or right from the center of the screen. Finally, the participants were asked to perform the rating procedure.

\section{Data Analysis}

The purpose-written software package Begaze (SMI, Berlin) was used for the analysis of eye movement data. For the saccade task, two parameters were calculated to assess task performance: the rates of errors in pro- and antisaccades and the latencies of correct pro- and antisaccades. The error rates in antisaccades (i.e., erratic prosaccades) are regarded as an index of a lack of volitional inhibition of reflexive attention (Hutton \& Ettinger, 2006). The detection of saccades was based on the criteria of minimum amplitude $\left(1.5^{\circ}\right)$ and minimum velocity $(30 \% \mathrm{sec})$. Saccades with an onset shorter than $80 \mathrm{msec}$ after the appearance of the target were not considered as triggered by the stimulus and were therefore classified as anticipatory saccades (Reuter, Jäger, Bottlender, \& Kathmann, 2007). Antisaccade errors (erratic prosaccades) were counted when the participant performed a primary saccade with an amplitude $>3^{\circ}$ toward the target picture (on antisaccade trials); prosaccadic errors were counted when participants performed a primary saccade in the opposite direction of the peripheral target picture. Latencies of antisaccades and prosaccades were determined relative to the stimulus picture onset. Trials containing anticipatory saccades, recording artifacts, or blinks were excluded from analysis. Percentages of reflexive prosaccades and of antisaccades were computed relative to the number of valid trials in each participant. Mean error rates and latencies were afterward analyzed by repeated measures ANOVAs, with group as the between-subjects factor (3: LSA vs. controls vs. HSA), and with task (2: prosaccade vs. antisaccade) and emotion (5: angry vs. happy vs. fearful vs. sad vs. neutral facial expression) as within-subjects factors.

Affective ratings were also analyzed by repeated measures ANOVAs with group as the between-subjects factor (3: LSA vs. controls vs. HSA) and emotion (5: angry vs. happy vs. fearful vs. sad vs. neutral) as the within-subjects factor. ${ }^{1}$

For all analyses, the $\alpha$ level was set to $5 \%$. If necessary, the Greenhouse-Geisser correction for violations of sphericity was used and the Greenhouse-Geisser epsilon $(\mathrm{GG}-\varepsilon)$ is reported. Significant interactions were followed up with ANOVAs and post hoc $t$ tests using Bonferroni adjustment of $\alpha$ level. Furthermore, for all analyses, effect sizes are reported as $\eta_{\mathrm{p}}^{2}$.

\section{RESULTS}

\section{Error Rates}

Error rates were higher for the antisaccade task than for the prosaccade task $\left[F(1,58)=71.2, p<.001, \eta_{\mathrm{p}}^{2}=.55\right]$. In addition, groups differed in their error rates $[F(2,58)=$ $\left.3.1, p=.050, \eta_{\mathrm{p}}^{2}=.10\right]$, but this effect was expressed differently in the two tasks, as the significant interaction of task $\times$ group indicates $\left[F(2,58)=5.5, p=.005, \eta_{\mathrm{p}}^{2}=\right.$ .16] (see Figure 2).

To follow up this interaction, separate ANOVAs for each task were conducted. In the prosaccade task, there were no differences found in error rates between the three groups. However, in the antisaccade task, the error rate differed between the groups, as the significant main effect group revealed $\left[F(1,58)=4.6, p=.014, \eta_{\mathrm{p}}^{2}=.14\right]$. Post hoc comparisons between the groups (Bonferroni adjusted) showed that the HSA group made significantly more errors than did the controls $(p=.011)$, whereas there were no differences found in the performance between the HSA and LSA groups, and the LSA group and controls, respectively $(p s>.37)$. Error rates were modulated by emotion neither in the three groups nor overall, as the nonsignificant three-way interaction (task $\times$ group $\times$ emotion) and two-way interaction (emotion $\times$ task) revealed $\left[F(8,232)<1, p=.70, \eta_{\mathrm{p}}^{2}=.02\right.$, and $F(4,232)<1, p=$ $.41, \eta_{\mathrm{p}}^{2}=.02$, respectively]. Error rates in the three groups in each experimental condition are given in Table 2 .

\section{Latencies of Correct Pro- and Antisaccades}

As was expected, the latencies were significantly shorter for the prosaccades than for the antisaccades $[F(1,58)=$ 157.6, $\left.p<.001, \eta_{\mathrm{p}}^{2}=.73\right]$. However, this effect was further qualified by an interaction with the emotional content of the pictures (task $\times$ emotion) $[F(4,232)=6.7, \mathrm{GG}-\varepsilon=.40$, $\left.p=.004, \eta_{\mathrm{p}}^{2}=.10\right]$. Post hoc ANOVAs were conducted for each task separately. They revealed that this interaction was due to a significant main effect of emotion in the prosaccade

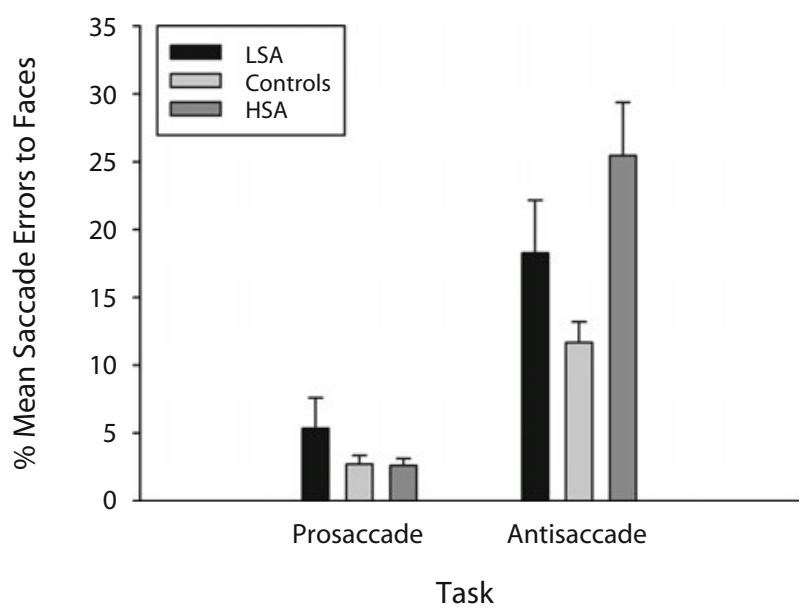

Figure 2. Mean percentage of errors in the pro- and antisaccade task collapsed over all facial expressions in the low-social-anxiety (LSA), control, and high-social-anxiety (HSA) groups. 
Table 2

Mean Error Rates (in Percentages) and Standard Deviations for Pro- and Antisaccades in Response to Facial Expressions for Each of the Three Groups

\begin{tabular}{|c|c|c|c|c|c|c|c|c|c|c|c|c|}
\hline \multirow[b]{3}{*}{ Facial Expression } & \multicolumn{6}{|c|}{ Prosaccade } & \multicolumn{6}{|c|}{ Antisaccade } \\
\hline & \multicolumn{2}{|c|}{ LSA } & \multicolumn{2}{|c|}{ Controls } & \multicolumn{2}{|c|}{ HSA } & \multicolumn{2}{|c|}{ LSA } & \multicolumn{2}{|c|}{ Controls } & \multicolumn{2}{|c|}{ HSA } \\
\hline & $M$ & $S D$ & $M$ & $S D$ & $M$ & $S D$ & $M$ & $S D$ & $M$ & $S D$ & $M$ & $S D$ \\
\hline Fearful & 3.6 & 12.2 & 2.9 & 4.2 & 1.8 & 3.8 & 18.4 & 20.7 & 12.1 & 10.1 & 25.3 & 23.0 \\
\hline Angry & 5.6 & 11.8 & 3.5 & 6.2 & 3.0 & 6.2 & 18.5 & 22.2 & 12.6 & 9.5 & 29.8 & 22.9 \\
\hline Happy & 7.0 & 10.7 & 1.7 & 3.6 & 1.7 & 3.5 & 18.2 & 20.1 & 11.7 & 9.1 & 21.3 & 18.4 \\
\hline Sad & 5.9 & 10.2 & 3.0 & 7.1 & 3.9 & 5.9 & 23.7 & 29.2 & 12.5 & 11.7 & 25.7 & 23.1 \\
\hline Neutral & 4.7 & 13.5 & 2.4 & 4.6 & 2.7 & 4.2 & 12.6 & 15.1 & 9.5 & 10.5 & 25.1 & 22.3 \\
\hline
\end{tabular}

Note-LSA, low-social-anxiety group; HSA, high-social-anxiety group.

$\operatorname{task}\left[F(4,240)=12.2, p<.001, \eta_{\mathrm{p}}^{2}=.17\right]$. Simple contrasts showed that all participants had shortened latencies for prosaccades in response to fearful $(M=222.2 \mathrm{msec}, S D=$ $79.6)$ as compared with angry $(M=245.4, S D=85.9)$, happy $(M=244.4, S D=88.6), \operatorname{sad}(M=246.2, S D=$ $89.7)$, and neutral $(M=249.9, S D=91.6)$ facial expressions (all contrasts, $F>23, p<.001$ ). For the antisaccade task, this main effect of emotion was marginally significant $\left[F(4,240)=2.4, p=.061, \eta_{\mathrm{p}}^{2}=.05\right]$. There was a tendency toward prolonged latencies for fearful $(M=320.5, S D=$ $127.5)$ as compared with angry $(M=292.1, S D=97.3)$, happy $(M=295.4, S D=94.9)$, $\operatorname{sad}(M=299.2, S D=$ $90.0)$, and neutral $(M=292.5, S D=90.8)$ facial expressions (all contrasts, $F>2.5, p<.089$ ).

No group differences were found for latencies of correct pro- and antisaccades (see Table 3). Neither the two-way interaction of group $\times$ task nor the two-way interaction of group $\times$ emotion approached significance $[F(2,58)<1$, $p=.50, \eta_{\mathrm{p}}^{2}=.02$, and $F(8,232)<1, p=.67, \eta_{\mathrm{p}}^{2}=.03$, respectively].

\section{Controlling for Effects of General State and Trait Anxiety (ANCOVA)}

Because the groups differed in their scores on the Spielberger scales of state and trait anxiety, all the statistical analyses of eye movement data were repeated using these individual scores as covariates. By showing the same significant group $\times$ task interaction in error rates $[F(2,56)=$ $\left.6.1, p=.004, \eta_{\mathrm{p}}^{2}=.18\right]$, the findings from the ANCOVAs corroborated those from the ANOVAs. Thus, the effect may not have been due to actual and trait anxiety, but might be explained by the amount of social anxiety in the groups.

\section{DISCUSSION}

To our knowledge, the present study is the first to examine the predictions of the attentional control theory in social anxiety by means of an emotional saccade task. Overall, the results from other standard saccade tasks could be replicated with prolonged latencies of correct antisaccades in comparison with latencies of correct prosaccades and with more errors in the antisaccade task than in the prosaccade task. Furthermore, the present results showed overall prolonged latencies for correct antisaccades and shortened latencies for correct prosaccades in response to fearful faces, which indicates that these facial expressions may attract attention in all participants regardless of social anxiety. Thus, fearful expressions seem to be very powerful in attracting attention and hard to avoid voluntarily in humans. This finding is in line with those of neuroimaging studies showing the most powerful, most consistent, and quickest brain activations in fear-related structures, such as the amygdala, in response to fearful facial expressions (Killgore \& Yurgelun-Todd, 2005; Stein, Goldin, Sareen, Zorrilla, \& Brown, 2002; Straube, Kolassa, Glauer, Mentzel, \& Miltner, 2004).

Interestingly, during the antisaccade task, high socially anxious participants showed more errors in response to all facial expressions. As a consequence, a more general phenomenon of attention-control-related deficit in social anxiety may account for these findings. The data probably indicate a lowered executive functioning and attentional control in response to facial expressions. However, it is important to note that in the present study this deficit is

Table 3

Mean Latencies (in Milliseconds) and Standard Deviations for Correct Pro- and Antisaccades in Response to Facial Expressions for Each of the Three Groups

\begin{tabular}{|c|c|c|c|c|c|c|c|c|c|c|c|c|}
\hline \multirow[b]{3}{*}{ Facial Expression } & \multicolumn{6}{|c|}{ Prosaccade } & \multicolumn{6}{|c|}{ Antisaccade } \\
\hline & \multicolumn{2}{|c|}{ LSA } & \multicolumn{2}{|c|}{ Controls } & \multicolumn{2}{|c|}{ HSA } & \multicolumn{2}{|c|}{ LSA } & \multicolumn{2}{|c|}{ Controls } & \multicolumn{2}{|c|}{ HSA } \\
\hline & $M$ & $S D$ & $M$ & $S D$ & $M$ & $S D$ & $M$ & $S D$ & $M$ & $S D$ & $M$ & $S D$ \\
\hline Fearful & 219.3 & 86.8 & 233.6 & 79.7 & 213.0 & 74.3 & 340.4 & 225.1 & 311.9 & 102.4 & 309.6 & 80.1 \\
\hline Angry & 247.5 & 90.9 & 251.1 & 89.3 & 237.3 & 80.7 & 277.7 & 99.4 & 303.9 & 101.1 & 294.2 & 94.1 \\
\hline Нарру & 240.7 & 92.7 & 250.4 & 86.5 & 241.8 & 90.9 & 281.1 & 104.1 & 305.2 & 95.1 & 299.4 & 88.1 \\
\hline $\mathrm{Sad}$ & 249.8 & 105.0 & 252.2 & 83.4 & 236.3 & 83.2 & 287.5 & 96.7 & 306.4 & 94.4 & 303.2 & 81.4 \\
\hline Neutral & 260.1 & 107.9 & 249.6 & 77.7 & 240.1 & 91.1 & 286.7 & 93.1 & 303.9 & 94.4 & 286.3 & 88.3 \\
\hline
\end{tabular}

Note-LSA, low-social-anxiety group; HSA, high-social-anxiety group. 
not due to higher state or trait anxiety of the high socially anxious participants, as the additional analyses using these anxiety scores as covariates revealed.

Neuroimaging studies have indeed suggested that anxiety is associated with reduced top-down control over threat-related distractors (Bishop et al., 2004). Participants with high-state anxiety showed less activity in the anterior cingulate cortex and prefrontal cortex in expectance of threat-related distractors (fearful faces) than did low-anxious persons. The authors interpreted this reduced recruitment of brain areas, which seem to be associated with cognitive control (Carter et al., 1998; MacDonald, Cohen, Stenger, \& Carter, 2000), as an index of disrupted attentional control over threat-related information in anxiety. The antisaccade performance is considered to depend on the ability to adequately maintain the task instruction while inhibiting the reflexive response to the task-irrelevant stimuli (Hutton \& Ettinger, 2006). The relevant brain areas include the anterior cingulate and the prefrontal cortex (Ettinger et al., 2008; Müri et al., 1998; O'Driscoll et al., 1995). Thus, the deficits in antisaccade performance found in the present study support the notion that socially anxious persons show deficits in their cognitive control of attention. Note, however, that in this standard antisaccade task the process of response inhibition and volitional response generation are likely to occur simultaneously (Massen, 2004). Thus, the temporal dissociation of the inhibition of the prepotent response on the one hand, and the generation of a volitional saccadic response on the other hand, cannot be tested. Recently, it has been shown that in a delayed antisaccade task, which introduces a temporal gap between the presentation of the target and an auditory signal to perform the antisaccade, prefrontal areas (VLPFC and DLPFC) showed comparable activations in both phases of the task (Ettinger et al., 2008). This finding points at a more general role of these areas in the volitional control of eye movements.

In another recent study on the effects of anxiety on task switching, a lack of anxiety-related effects on saccade accuracy was observed (Ansari, Derakshan, \& Richards, 2008). This observation was interpreted as a reduced efficiency (the amount of processing resources needed to perform the task) in anxiety, whereas effectiveness (the competence in doing the task) seemed to be spared. In our present study, however, the opposite pattern was observed: a diminished effectiveness in terms of more errors in high socially anxious people, but no group differences in the efficiency in terms of latencies of correct pro- and antisaccades. Whereas the stimulus used by Ansari and colleagues had no emotional relevance itself (dots), the display of a human face might have a special salience for socially anxious persons regardless of the emotional expression. Using electrocortical measures, it has been shown that faces per se may be attended more by socially anxious individuals and social phobic patients, as overall enlarged P100 amplitudes to faces has indicated (Kolassa \& Miltner, 2006; Mühlberger et al., 2009). Furthermore, it has been shown that not only angry facial expressions seem to attract attention and lead to elaborated processing in socially anxious individuals (Garner, Mogg, \& Bradley, 2006a; Moser, Duval, \& Simons, 2006; Wieser et al., 2009). As Gentili et al. (2008) showed, this preferential processing of facial expressions in social phobia is also accompanied by a decreased activation in prefrontal areas, which points at weakened volitional attentional control and might be the reason for hypervigilance and abnormal scanning of faces in social anxiety. Altogether, these findings are consistent with our results of an overall impaired inhibition of the probably prepotent orienting response to social stimuli indicated by the heightened error rate in the antisaccade task in socially anxious individuals.

There are various ways in which future research might address the limitations of the present investigation. Although significant effects were not observed in the performance parameters, the inclusion of a low socially anxious sample based on the lowest $10 \%$ of a questionnaire may be critical, since this sample may include low-motivated individuals. Also, because of the missing nonsocial control stimulus in our present study, future research has to show whether the deficit in high socially anxious participants is a general deficit in attention control or whether it is specific concerning facial stimuli. Consequently, future research should include nonsocial stimuli such as scrambled faces or houses. Furthermore, it has to be shown whether high socially anxious persons perform worse in any cognitive task or whether these deficits are specific in tasks requiring attentional control and the recruitment of prefrontal areas. This might already be achieved to some extent in a delayed pro- or antisaccade task (Reuter \& Kathmann, 2004) in which, on the one hand, the inhibition of unwanted responses, and on the other hand, the generation of a volitional response (PierrotDeseilligny, Rivaud, Gaymard, \& Agid, 1991) can be dissociated. Furthermore, the attentional control in response to facial expressions has to be investigated in clinically diagnosed patients with social phobia.

To sum up, the present study is the first to investigate attentional control in socially anxious participants in response to facial expressions by means of the antisaccade task. Our findings go in line with previous neuroimaging results (Gentili et al., 2008) by showing a diminished volitional inhibition of prepotent responses to facial expressions in socially anxious participants. The emotional saccade task seems to be an excellent research tool for further investigations of the attentional control theory in both anxiety and other mood disorders.

\section{AUTHOR NOTE}

We thank Anne Springmann and Christian Baumann for their help in data collection. This work was supported by Grant MU2299-1/2 by the Deutsche Forschungsgemeinschaft (DFG). Address correspondence to A. Mühlberger, Department of Psychology, Biological Psychology, Clinical Psychology, and Psychotherapy, University of Würzburg, Marcusstr. 9-11, D-97070 Würzburg, Germany (e-mail: muehlberger@ psychologie.uni-wuerzburg.de).

\section{REFERENCES}

Ansari, T. L., Derakshan, N., \& Richards, A. (2008). Effects of anxiety on task switching: Evidence from the mixed antisaccade task. $C o g$ nitive, Affective, \& Behavioral Neuroscience, 8, 229-238.

Bar-Haim, Y., Lamy, D., Pergamin, L., BaKermans-Kranenburg, 
M. J., \& VAN IJZEndoorn, M. H. (2007). Threat-related attentional bias in anxious and nonanxious individuals: A meta-analytic study. Psychological Bulletin, 133, 1-24.

Bell, A. H., Everling, S., \& Munoz, D. P. (2000). Influence of stimulus eccentricity and direction on characteristics of pro- and antisaccades in non-human primates. Journal of Neurophysiology, 84, 2595-2604.

Birbaumer, N., Grodd, W., Diedrich, O., Klose, U., Erb, M., Lotze, M., ET AL. (1998). fMRI reveals amygdala activation to human faces in social phobics. NeuroReport, 9, 1223-1226.

BisHOP, S. J. (2007). Neurocognitive mechanisms of anxiety: An integrative account. Trends in Cognitive Sciences, 11, 307-316.

Bishop, S. J., Duncan, J., Brett, M., \& Lawrence, A. D. (2004). Prefrontal cortical function and anxiety: Controlling attention to threatrelated stimuli. Nature Neuroscience, 7, 184-188.

Broerse, A., Crawford, T. J., \& den Boer, J. A. (2001). Parsing cognition in schizophrenia using saccadic eye movements: A selective overview. Neuropsychologia, 39, 742-756.

Carter, C. S., Braver, T. S., Barch, D. M., Botvinick, M. M., Noll, D., \& Cohen, J. D. (1998). Anterior cingulate cortex, error detection, and the online monitoring of performance. Science, $\mathbf{2 8 0}$, 747-749.

Coles, M. E., Heimberg, R. G., \& Schofield, C. A. (2008). Interpretation of facial expressions and social anxiety: Specificity and source of biases. Cognition \& Emotion, 22, 1159-1173.

Crawford, T. J., Bennett, D., Lekwuwa, G., Shaunak, S., \& DEAKIN, J. F. (2002). Cognition and the inhibitory control of saccades in schizophrenia and Parkinson's disease. Progress in Brain Research, 140, 449-466.

Derakshan, N., Ansari, T. L., Hansard, M., Shoker, L., \& Eysenck, M. W. (2009). Anxiety, inhibition, efficiency, and effectiveness. Experimental Psychology, 56, 48-55.

Ekman, P., \& Friesen, W. V. (1976). Pictures of facial affect. Palo Alto, CA: Consulting Psychologists Press.

Ekman, P., \& Friesen, W. V. (1978). Facial action coding system: Investigator's guide Part I. Palo Alto, CA: Consulting Psychologists Press.

Ettinger, U., fFytche, D. H., Kumari, V., Kathmann, N., ReuTer, B., Zelaya, F., \& Williams, S. C. R. (2008). Decomposing the neural correlates of antisaccade eye movements using event-related fMRI. Cerebral Cortex, 18, 1148-1159.

EverLing, S., \& Fischer, B. (1998). The antisaccade: A review of basic research and clinical studies. Neuropsychologia, 36, 885-899.

Eysenck, H. J., \& Eysenck, S. B. G. (1991). Manual of the Eysenck Personality Scales (EPS adult). London: Hodder and Stoughton.

Eysenck, M. W., Derakshan, N., Santos, R., \& Calvo, M. G. (2007). Anxiety and cognitive performance: Attentional control theory. Emotion, 7, 336-353.

FydRICH, T. (2002). SPAI-Soziale Phobie und Angst Inventar. In E. Brähler, J. Schumacher, \& B. Strauß (Eds.), Diagnostische Verfahren in der Psychotherapie (pp. 335-338). Göttingen: Hogrefe.

Garner, M., MogG, K., \& Bradley, B. P. (2006a). Fear-relevant selective associations and social anxiety: Absence of a positive bias. Behaviour Research \& Therapy, 44, 201-217.

Garner, M., MogG, K., \& Bradley, B. P. (2006b). Orienting and maintenance of gaze to facial expressions in social anxiety. Journal of Abnormal Psychology, 115, 760-770.

Gentili, C., Gobbini, M. I., Ricciardi, E., Vanello, N., Pietrini, P., Haxby, J. V., \& Guazzelli, M. (2008). Differential modulation of neural activity throughout the distributed neural system for face perception in patients with social phobia and healthy subjects. Brain Research Bulletin, 77, 286-292.

Gerdes, A. B. M., Alpers, G. W., \& Pauli, P. (2008). When spiders appear suddenly: Spider-phobic patients are distracted by task-irrelevant spiders. Behaviour Research \& Therapy, 46, 174-187.

Hallett, P. E. (1978). Primary and secondary saccades to goals defined by instructions. Vision Research, 18, 1279-1296.

Horley, K., Williams, L. M., Gonsalvez, C., \& Gordon, E. (2003). Social phobics do not see eye to eye: A visual scanpath study of emotional expression processing. Journal of Anxiety Disorders, 17, 33-44.

Hutton, S. B., \& EtTinger, U. (2006). The antisaccade task as a research tool in psychopathology: A critical review. Psychophysiology, 43, 302-313.

JaZbec, S., McClure, E., Hardin, M., Pine, D. S., \& Ernst, M. (2005).
Cognitive control under contingencies in anxious and depressed adolescents: An antisaccade task. Biological Psychiatry, 58, 632-639.

Killgore, W. D., \& Yurgelun-TodD, D. A. (2005). Social anxiety predicts amygdala activation in adolescents viewing fearful faces. NeuroReport, 16, 1671-1675.

Kissler, J., \& KeIL, A. (2008). Look-don't look! How emotional pictures affect pro- and anti-saccades. Experimental Brain Research, $\mathbf{1 8 8}, 215-222$

Klein, C. H., Raschke, A., \& Brandenbusch, A. (2003). Development of pro- and antisaccades in children with attention-deficit hyperactivity disorder (ADHD) and healthy controls. Psychophysiology, 40, 17-28.

Kolassa, I.-T., Kolassa, S., Musial, F., \& Miltner, W. H. R. (2007). Event-related potentials to schematic faces in social phobia. Cognition \& Emotion, 21, 1721-1744.

Kolassa, I.-T., \& Miltner, W. H. R. (2006). Psychophysiological correlates of face processing in social phobia. Brain Research, 1118, 130-141.

Krohne, H. W., Egloff, B., Kohlmann, C.-W., \& Tausch, A. (1996). Untersuchungen mit einer deutschen Version der "Positive and Negative Affect Schedule" (PANAS). Diagnostica, 42, 139-156.

Laux, L., Glanzmann, P., Schaffner, P., \& Spielberger, C. D. (1981). Das State-Trait-Angstinventar. Weinheim: Beltz Test.

LEARY, M. R. (1983). A brief version of the fear of negative evaluation scale. Personality \& Social Psychology Bulletin, 9, 371-375.

MacDonald, A. W., Cohen, J. D., Stenger, V. A., \& Carter, C. S. (2000). Dissociating the role of the dorsolateral prefrontal and anterior cingulate cortex in cognitive control. Science, 288, 1835-1838.

Massen, C. (2004). Parallel programming of exogenous and endogenous components in the antisaccade task. Quarterly Journal of Experimental Psychology, 57A, 475-498.

Miltner, W. H. R., Krieschel, S., Hecht, H., Trippe, R., \& Weiss, T. (2004). Eye movements and behavioral responses to threatening and nonthreatening stimuli during visual search in phobic and nonphobic subjects. Emotion, 4, 323-339.

Moser, J. S., Duval, E., \& Simons, R. F. (2006). Friend, foe, or I don't know? Electrophysiological evidence of enhanced processing of neutral facial expressions in social phobia [Abstract]. Psychophysiology, 43, S69.

Mühlberger, A., Wieser, M. J., Herrmann, M. J., Weyers, P., Tröger, C., \& PAuli, P. (2009). Early cortical processing of natural and artificial emotional faces differs between lower and higher socially anxious persons. Journal of Neural Transmission, 116, 735-746. doi:10.1007/s00702-008-0108-6

Müri, R. M., Heid, O., Nirkko, A. C., Ozdoba, C., Felblinger, J., Schroth, G., \& Hess, C. W. (1998). Functional organization of saccades and antisaccades in the frontal lobe in humans: A study with echo planar functional magnetic resonance imaging. Journal of Neurology, Neurosurgery, \& Psychiatry, 65, 374-377.

O’Driscoll, G. A., Alpert, N. M., Matthysse, S. W., Levy, D. L., RaUCH, S. L., \& Holzman, P. S. (1995). Functional neuroanatomy of antisaccade eye movements investigated with positron emission tomography. Proceedings of the National Academy of Sciences, 92 , 925-929.

Pashler, H., Johnston, J. C., \& Ruthruff, E. (2001). Attention and performance. Annual Review of Psychology, 52, 629-651.

Pierrot-Deseilligny, C., Rivaud, S., Gaymard, B., \& Agid, Y. (1991). Cortical control of reflexive visually-guided saccades. Brain, 114, 1473-1485.

Posner, M. I., \& Petersen, S. E. (1990). The attention system of the human brain. Annual Review of Neuroscience, 13, 25-42.

Reuter, B., JäGer, M., Bottlender, R., \& Kathmann, N. (2007). Impaired action control in schizophrenia: The role of volitional saccade initiation. Neuropsychologia, 45, 1840-1848.

Reuter, B., \& Kathmann, N. (2004). Using saccade tasks as a tool to analyze executive dysfunctions in schizophrenia. Acta Psychologica, 115, 255-269.

RiNCK, M., \& BECKER, E. S. (2006). Spider fearful individuals attend to threat, then quickly avoid it: Evidence from eye movements. Journal of Abnormal Psychology, 115, 231-238.

RuCH, W. (1999). Die revidierte Fassung des Eysenck Personality Questionnaire und die Konstruktion des deutschen (EPQ-R bzw. EPQ-RK) [The Eysenck Personality Questionnaire-Revised and the construction 
of the German standard and short versions (EPQ-R and EPQ-RK)]. Zeitschrift für Differentielle und Diagnostische Psychologie, 20, 1-24. Spencer-Smith, J., Wild, H., Innes-Ker, A. H., Townsend, J., Duffy, C., Edwards, C., ET AL. (2001). Making faces: Creating three-dimensional parameterized models of facial expression. Behavior Research Methods, Instruments, \& Computers, 33, 115-123.

Spielberger, C. D., Gorsuch, R. L., \& Lushene, R. E. (1970). StateTrait Anxiety Inventory. Palo Alto, CA: Consulting Psychologists Press.

Stein, M. B., Goldin, P. R., Sareen, J., Zorrilla, L. T. E., \& Brown, G. G. (2002). Increased amygdala activation to angry and contemptuous faces in generalized social phobia. Archives of General Psychiatry, 59, 1027-1034.

Straube, T., Kolassa, I. T., Glauer, M., Mentzel, H. J., \& Miltner, W. H. (2004). Effect of task conditions on brain responses to threatening faces in social phobics: An event-related functional magnetic resonance imaging study. Biological Psychiatry, 56, 921-930.

Straube, T., Mentzel, H. J., \& Miltner, W. H. R. (2005). Common and distinct brain activation to threat and safety signals in social phobia. Neuropsychobiology, 52, 163-168.

Turk, C. L., Heimberg, R. G., Orsillo, S. M., Holt, C. S., Gitow, A., Street, L. L., ET AL. (1998). An investigation of gender differences in social phobia. Journal of Anxiety Disorders, 12, 209-223.

Turner, S. M., Beidel, D. C., Dancu, C. V., \& Stanley, M. A. (1989). An empirically derived inventory to measure social fears and anxiety: The Social Phobia and Anxiety Inventory (SPAI). Psychological Assessment, 1, 35-40.

Watson, D., Clark, L. A., \& Tellegen, A. (1988). Development and validation of brief measures of positive and negative affect: The PANAS scales. Journal of Personality \& Social Psychology, 54, 1063-1070.

Wieser, M. J., Pauli, P., Weyers, P., Alpers, G. W., \& Mühlberger, A. (2009). Fear of negative evaluation and the hypervigilance-avoidance hypothesis: An eye-tracking study. Journal of Neural Transmission, 116, 717-723. doi:10.1007/s00702-008-0101-0

\section{NOTE}

1. The results of the ratings are presented in a more detailed way elsewhere, as yet unpublished. Altogether, faces were rated as was expected: For the valence ratings, there was a significant main effect of emotion $\left[F(4,228)=488.3, \mathrm{GG}-\varepsilon=.58, p<.001, \eta_{\mathrm{p}}^{2}=.90\right]$. Simple contrast analyses with neutral faces as a reference category revealed that negative facial expressions were rated as more negative than were neutral and happy faces (all $p \mathrm{~s}<.001$ ), and happy faces were rated as more positive than neutral ones $(p<.001)$. Groups differed in their overall ratings of the facial expressions $\left[F(2,57)=3.8, p=.03, \eta_{\mathrm{p}}^{2}=.12\right]$. Post hoc comparisons revealed that this effect was due to a significant difference between HSA and LSA only ( $p=.03$ ) (Bonferroni adjusted). Thus, the HSA group rated facial expressions regardless of emotion as more negative than did the LSA group. Regarding the arousal ratings, the expected significant effect of emotion occurred $\left[F(4,228)=64.4\right.$, GG- $\left.\varepsilon=.60, p<.001, \eta_{\mathrm{p}}^{2}=.53\right]$. Simple contrast analyses showed that all emotional facial expressions were rated as more arousing than the neutral ones (all $p \mathrm{~s}<.001$ ).

(Manuscript received August 22, 2008; revision accepted for publication March 21, 2009.) 